\title{
The Dynamical Assessment of Inflammatory Biomarkers in Predicting the Outcome of Septic Patients and the Response to Antimicrobial Therapy
}

\author{
Alina Orfanu1,2*, Victoria Aramă1,2, Cristina Popescu ${ }^{1,2}$, Cătălin Tilişcan ${ }^{1,2}$, Adrian Streinu- \\ Cercel $^{1,2}$, Ştefan Sorin Aramă ${ }^{1,2}$ \\ 1 National Institute for Infectious Diseases "Prof. Dr. Matei Bals", Bucharest \\ 2 University of Medicine and Pharmacy "Carol Davila", Bucharest
}

\begin{abstract}
Aims: To evaluate the kinetics of inflammatory biomarkers in septic patients in order to identify the most reliable predictor of unfavorable outcome. Methods: A prospective analysis of septic patients was performed. Median levels of neutrophil/lymphocyte count ratio, fibrinogen, C-reactive protein and procalcitonin were dynamically assessed and comparatively analyzed. Results: Seventy-seven patients were included. Descendent kinetic patterns were registered for all biomarkers, except C-reactive protein. At 24 hours, neutrophil/lymphocyte count ratio significantly decreased in $42.85 \%$ of cases, procalcitonin in $37.33 \%$, C-reactive protein in $16.12 \%$ and fibrinogen in $1.58 \%$ of cases. At 72 hours, procalcitonin decreased to one-half in $70 \%$ of cases and neutrophil/lymphocyte count ratio in $67.53 \%$ of cases. Conclusions: Neutrophil/lymphocyte count ratio and procalcitonin significantly decreased in the first 72 hours, while $\mathrm{C}$-reactive protein increased in the first 24 hours. The proportions of patients with major decrease of baseline values were higher for neutrophil/lymphocyte count ratio and procalcitonin.
\end{abstract}

Keywords: sepsis, biomarkers of inflammation, kinetic patterns, prognosis, therapy response

Received: 2 November 2019 / Accepted: 29 January 2020

\section{INTRODUCTION}

Despite the progression of bacterial isolation techniques over time, the identification of the infectious agent is still challenging in sepsis. The rates of positive blood cultures are between $30-50 \%$ in sepsis [1] and do not exceed $60-70 \%$ in septic shock $[2,3]$. Patients diagnosed with sepsis receive empiric broad-spectrum antibiotics at admission, followed by de-escalation of the antimicrobial regimen usually after few days, if the etiological agent is identified.

Even in cases with isolated pathogen, it lasts minimally 48-72 hours until the test results are obtained, delay which can be fatal for the patient.

In this situation, it is imperative to count on reliable instruments for an adequate monitoring of septic patients. Biomarkers of inflammation such as erythrocyte sedimentation rate (ESR), fibrinogen (FIB), C-reactive protein (CRP), procalcitonin (PCT) or haemogram parameters like neutrophil/lymphocyte count ratio (NLCR) or mean platelet volume (MPV) are traditionally used in cases of sepsis. Their usefulness is not only related to the early recognition of sepsis and to the assessment of disease severity at admission, but also to the prediction of the prognosis and the response to antimicrobial therapy [4].

PCT is a promising serum biomarker, characterized through high sensitivity and specificity for bacterial sepsis recognition [5]. Moreover, PCT levels increase in 3-4 hours in many bacterial systemic infections, achieve high levels in the first 24 hours [6] and rapidly decrease in patients receiving potent antibiotics [7]. CRP is characterized not only by lower sensitivity and specificity for sepsis diagnosis than PCT [8], but it presents a slower descendent pattern in comparison with PCT. After the contact with the pathogen, CRP starts to increase after 12-24 hours and remains elevated in the first 48-72 hours. This is the reason why in many situ- 
ations, the dynamical pattern of CRP presents a slow increasing in the first phase, followed by its maintaining at peak levels for 2-3 days [6]. Another useful biomarker in sepsis is fibrinogen, which produces a fibrin network in order to neutralize the bacterial invasion [9]. Similar to CRP, serum fibrinogen increases within few days after the onset of inflammatory process and reaches peak concentrations after 48 hours [10]. On the other hand, NLCR is characterized by an increasing in less than 6 hours after the exposure [11]. In a non-infectious population of patients who underwent orthopedic surgeries, postoperative NLCR levels registered a more rapid decrease than CRP.

The objective of the present study was to assess the kinetic patterns of NLCR, FIB, CRP and PCT in patients diagnosed with sepsis, in order to identify the most suitable instrument to monitor the outcome and the treatment response.

\section{MATERIAL AND METHODS}

\section{Study population and ethics}

We performed a prospective cross-sectional analysis of randomly-selected septic patients admitted in a tertiary-care department from National Institute for Infectious Diseases "Prof. Dr. Matei Balş", Bucharest, Romania. The enrollment included only patients who were hospitalized in a department of infectious diseases, while severe cases with multiple organ dysfunctions were directly admitted in the intensive care units. The recruitment of patients was performed between 2016 and 2019 and was based on sepsis-3 definition which involved the mandatory presence of a severe organ dysfunction [13]. The inclusion criteria were: Sepsis-related Organ Failure Assessment (SOFA) score at admission over 2 points and the presence of bacterial infection. The exclusion criteria were: age under 18 year-old, pregnancy and the evidence of noninfectious conditions responsible for an elevated SOFA score. Blood cultures, cultures from other biological fluids, serological tests or rapid molecular assays were performed in order to identify the infectious agent responsible for sepsis. The approval from the local ethics committee was obtained before the initiation of enrollment. An informed consent was signed by all patients who fulfilled the inclusion criteria. The research was performed according to Helsinki Declaration, revised in 2013.

\section{Study design}

The biomarkers of inflammation which were comparatively analyzed were NLCR, FIB, CRP and PCT. For the evaluation of biomarkers' dynamical patterns, three blood samples were collected: on admission in the infectious diseases department (day 0), after 24 hours (day 1) and after 72 hours (day 3 ). The median values of each biomarker were dynamically evaluated and the differences between the levels of serum biomarkers in dynamics were calculated. According to the kinetic pattern of each biomarker between every two collected samples (day 0 - day 1 , day 1 - day 3 and day 0 - day 3 ), the enrolled patients were divided in 4 groups: first group (1) - patients who registered an increasing or a minor decreasing (below 5\%) of serum concentrations of biomarkers between evaluated samples; second group (2) - patients who registered a decreasing between 5 and 20\%; third group (3) - patients with a decreasing between 20 and $50 \%$ and fourth group (4) - patients with a decreasing over $50 \%$ between two samples. The differences between proportions were assessed between every two biomarkers.

\section{Statistical analysis}

The analysis was performed using the Statistical Package for Social Sciences (SPSS), version 21.0 (SPSS Inc., Chicago, IL, USA). Continuous variables with normal distribution were presented as mean \pm standard deviation, while continuous variables with non-normal distribution were expressed as median and interquartile range (IQR). The distribution of variables was established according to Shapiro-Wilk and KolmogorovSmirnov tests. Wilcoxon Signed Ranks test was used in order to compare non-parametric variables. Sample proportions were compared using z-test. A value of $\mathrm{p}<0.05$ was considered statistically significant.

\section{口ESULTS}

Seventy-seven patients with a mean age of $59.91 \pm 17.09$ years met the inclusion criteria and were recruited during the study period. The gender ratio $\mathrm{M}: \mathrm{F}$ was $1: 2.08$. In $10.3 \%$ of patients, no associated chronic co-pathologies were identified. The most frequent associated comorbidities were: cardiovascular diseases $(47.8 \%)$, chronic neurological disorders (20.2\%), diabetes (17.3\%), chronic hepatitis or cirrhosis (8.6\%) and chronic renal disease (5.7\%). Patients with non-infectious conditions responsible for previously elevated levels of inflamma- 
tory biomarkers (malignancies, autoimmune disorders, recent surgeries or trauma) were not included into the study. In cases with identified etiology of sepsis, the mean period of time from admission until the bacteriological results was 48-72 hours. Polymerase chain reaction (PCR) assays had more rapid results in comparison with cultures or serological tests.

The median levels of NLCR, FIB, CRP and PCT are presented in Table 1, while the time courses of biomarkers and the differences between collected samples are exposed in Figure 1. NLCR, FIB and PCT registered descendent patterns and in cases of NLCR and PCT, the differences between samples were statistically significant. CRP levels increased after 24 hours from admission, but significantly decreased after 72 hours.
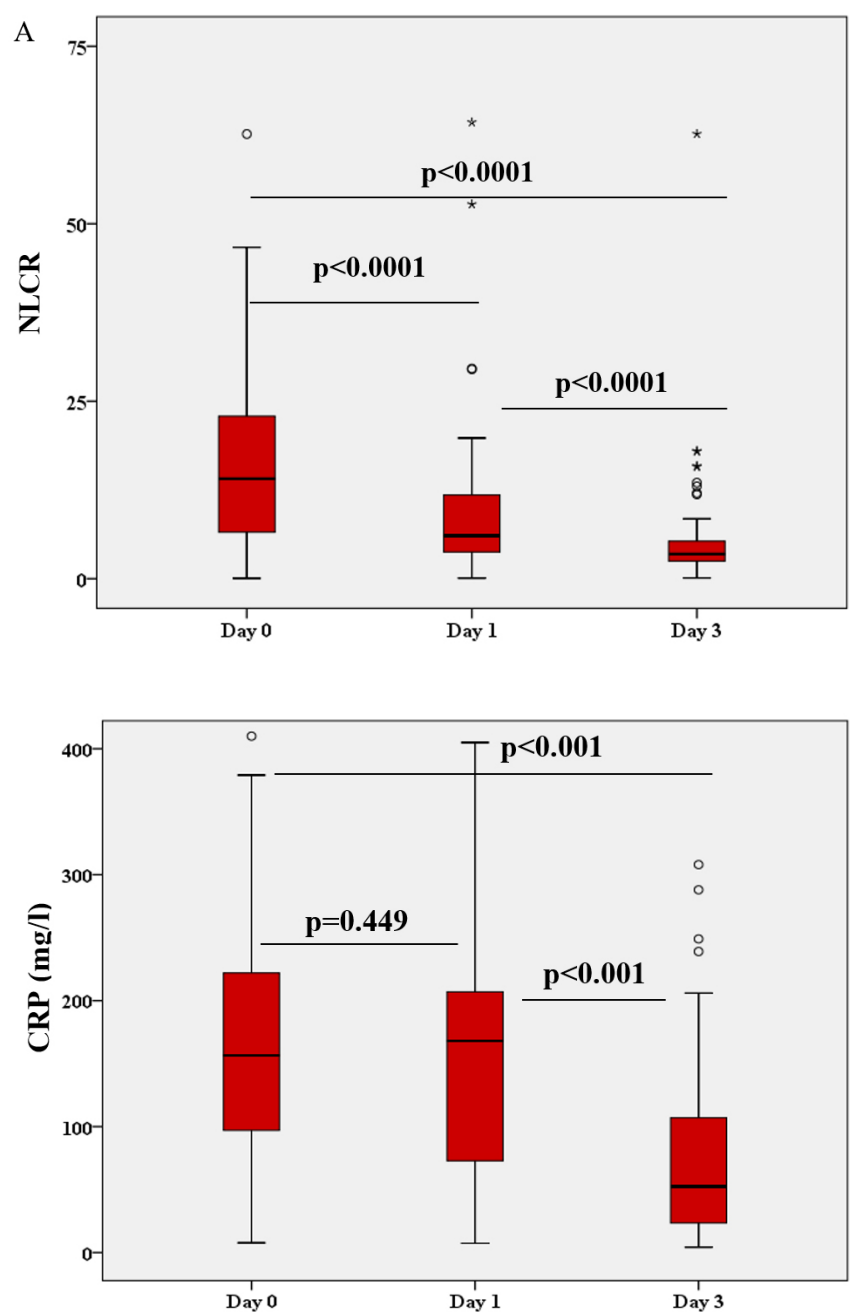

Table 1. The dynamical values of NLCR, FIB, CRP and PCT on days 0,1 and 3 . The results are presented as median (IQR).

$\begin{array}{lccc}\text { Variable } & \begin{array}{c}\text { Admission } \\ \text { (day 0) }\end{array} & \begin{array}{c}\text { After 24 } \\ \text { hours (day 1) }\end{array} & \begin{array}{c}\text { After } \mathbf{7 2} \text { hours } \\ \text { (day 3) }\end{array} \\ \text { NLCR } & 13.4 & 6.14 & 3.63 \\ (6 ; 22.8) & (3.7 ; 12.86) & (2.49 ; 5.98) \\ \text { FIB } & 606 & 564 & 474 \\ (\mathrm{mg} / \mathrm{dl}) & (475 ; 753) & (474 ; 730) & (385 ; 616) \\ \mathrm{CRP} & 157 & 169 & 58.4 \\ (\mathrm{mg} / \mathrm{l}) & (102 ; 232) & (73 ; 212) & (23.5 ; 109) \\ \mathrm{PCT} & 4.36 & 3.34 & 0.78 \\ (\mathrm{ng} / \mathrm{dl}) & (0.51 ; 22.71) & (0.36 ; 22.06) & (0.05 ; 4.77)\end{array}$

NLCR, Neutrophil/lymphocyte count ratio; FIB, fibrinogen; CRP, C-reactive protein; PCT, procalcitonin.
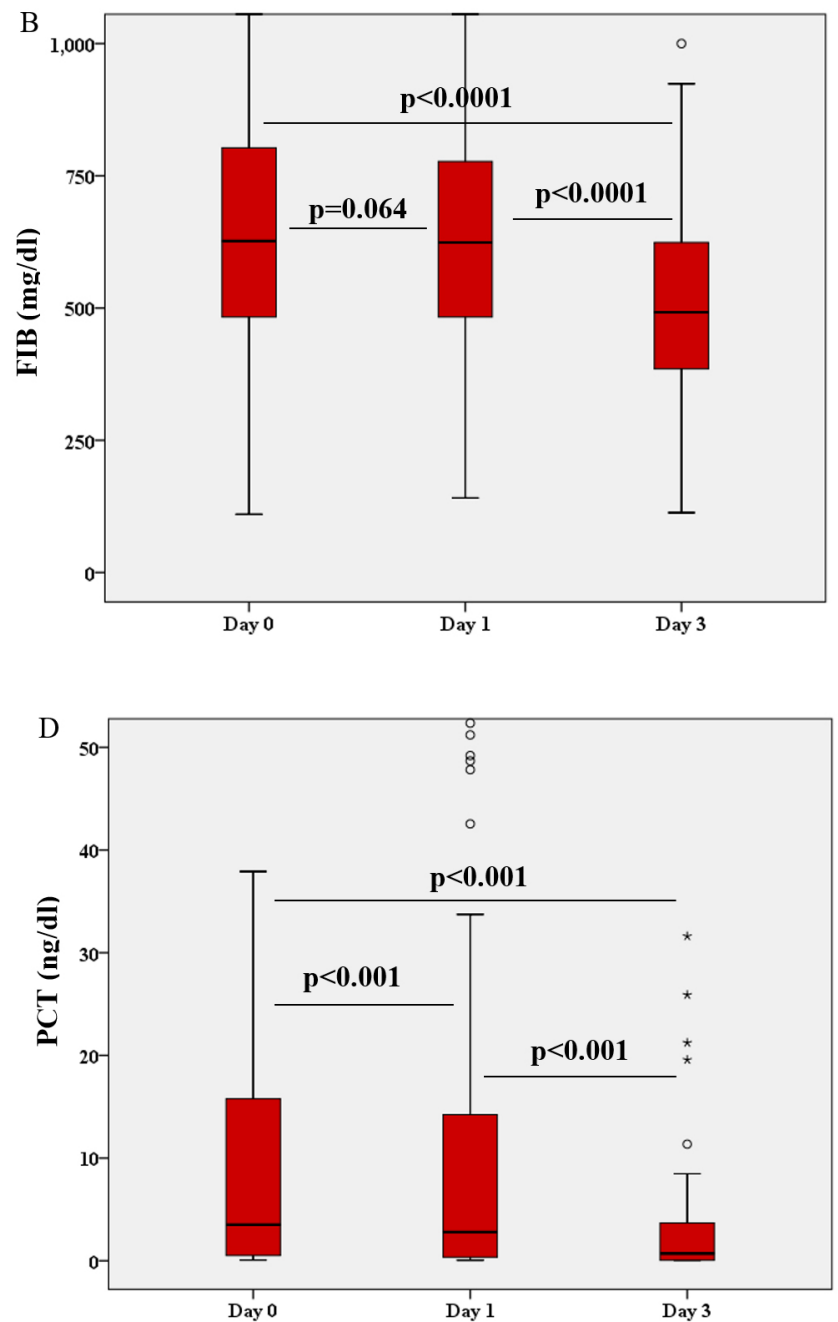

Fig. 1. The differences between the levels of serum biomarkers on days $\mathbf{0}, \mathbf{1}$ and $\mathbf{3}$. A. NLCR time course. Day $0-\mathrm{Day} 1$ difference, $p<0.0001$; Day 0 - Day 3 difference, $p<0.0001$; Day 1 - Day 3 difference, $p<0.0001$. B. FIB time course. Day $0-$ Day 1 difference, $p=0.064$; Day 0 - Day 3 difference, $p<0.0001$; Day 1 - Day 3 difference, $p<0.0001$. C. CRP time course. Day 0 - Day 1 difference, $p=0.449$; Day 0 - Day 3 difference, $p<0.001 ;$ Day 1 - Day 3 difference, $p<0.001$. C. PCT time course. Day 0 - Day 1 difference, $p<0.001$; Day 0 - Day 3 difference, $p=0.001$; Day 1 - Day 3 difference, $p<0.001$. NLCR, Neutrophil/ lymphocyte count ratio; FIB, fibrinogen; CRP, C-reactive protein; PCT, procalcitonin. 
For a more accurate comparison between serum biomarkers, their concentrations were dynamically evaluated, in order to identify their role in predicting a poor outcome. The first assessment was performed between the values on admission and 24 hours after admission (day 0 - day 1). At 24 hours, NLCR registered a decreasing of more than $50 \%$ of the initial values in $42.85 \%$ of cases, followed by PCT $(37.33 \%, \mathrm{p}=0.48)$, CRP (16.12\%, $\mathrm{p}=0.0007)$ and FIB (1.58\%, $\mathrm{p}<0.0001)$. Conversely, increased or decreased levels of less than $5 \%$ of the initial values appeared in case of FIB (52.38\%), followed by CRP (51.61\%, p=0.9313), PCT $(25.33 \%$, $\mathrm{p}=0.0011)$ and NLCR (22.07\%, $\mathrm{p}=0.0002)$. The other patients were enclosed in groups 2 or 3, with decreasing patterns, between 5 and $20 \%$ and between $20 \%$ and $50 \%$, respectively. The mentioned proportions of each biomarker are illustrated in Figure 2.

The second evaluation was performed between the levels of markers collected 24 hours and 72 hours after admission (day 1 - day 3 ). In the group of patients with major decreasing of serum biomarkers $(>50 \%$ of the values at 24 hours) were included: $62.66 \%$ of patients in case of PCT, in comparison with 55.45\% in case of CRP $(\mathrm{p}=0.4605), 44.15 \%$ in case of NLCR $(\mathrm{p}=0.0222)$ and $4.83 \%$ in case of FIB $(\mathrm{p}<0.0001)$. On the other hand, the levels of biomarkers increased or decreased with less than $5 \%$ in $32.25 \%$ of cases for FIB, in comparison with $23.37 \%$ of cases for NLCR ( $p=0.2429$ ), $13.33 \%$ of

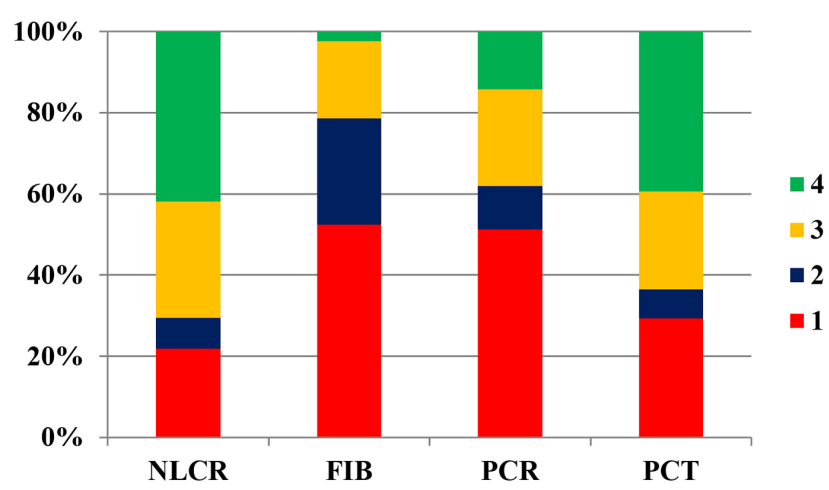

Fig. 2. Comparison of proportions of NLCR, FIB, CRP and PCT between days 0 and 1. 1 - patients who registered an increasing or a minor decreasing ( $<5 \%$ of the initial values) of serum biomarkers; 2 - patients who registered a decreasing of serum biomarkers between 5-20\%; 3 - patients who registered a decreasing of serum biomarkers between 20-50\%; 4 - patients who registered a major decreasing (>50\%) of serum biomarkers. NLCR, Neutrophil/lymphocyte count ratio; FIB, fibrinogen; CRP, C-reactive protein; $\mathrm{PCT}$, procalcitonin. cases for PCT $(\mathrm{p}=0.0077)$ and $11.29 \%$ of cases for CRP $(\mathrm{p}=0.0047)$. The proportions are presented in Figure 3.

The same analysis was performed to compare the proportions between admission and day 3 (day 0 - day 3). At 72 hours, PCT registered a decreasing over $50 \%$ of the initial values in $69.73 \%$ of patients, followed by NLCR (67.53\%, p=0.7694), CRP (58.2\%, p=0.1509) and FIB $(12 \%, \mathrm{p}<0.0001)$. In $13.15 \%$ of cases, PCT increased or decreased with less than $5 \%$, whereas the percent was higher for the other evaluated markers: $15.58 \%$ for NLCR ( $\mathrm{p}=0.6684), 26.86 \%$ for CRP $(\mathrm{p}=0.0392)$ and $38.66 \%$ for FIB $(\mathrm{p}=0.0003)$. The results are illustrated in Figure 4.

All patients included into the study received antimicrobial therapy, according to the identified or supposed etiological agent, to the primary site of infection and to the disease severity, appreciated at admission. Because the study was performed in a non-ICU department, only mild or moderate cases were included. In almost all cases, the outcome of patients was favorable under empirical therapy with broad-spectrum antibiotics. In one case of respiratory sepsis with unknown etiology, death occurred within 24 hours of admission.

De-escalation of antimicrobial therapy was performed in cases of identified pathogen. Even in cases with rapid descendent kinetic patterns of inflammatory biomarkers, the de-escalation could not be performed, in the absence of bacterial isolation.

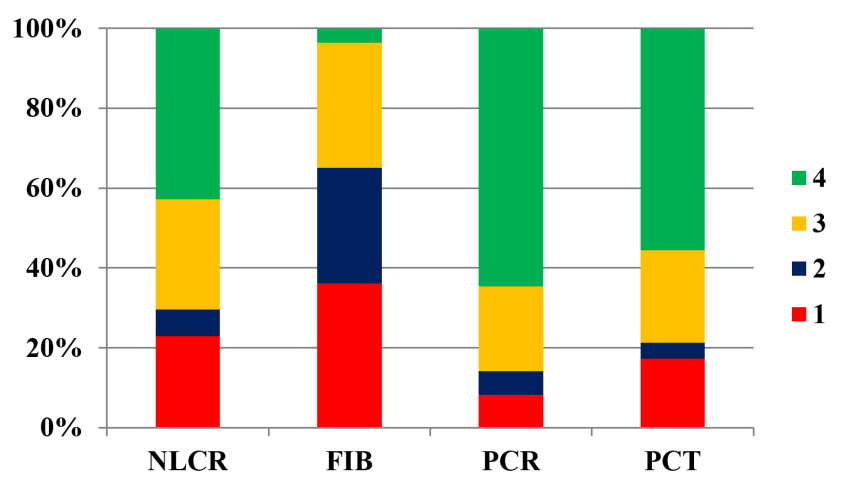

Fig. 3. Comparison of proportions of NLCR, FIB, CRP and PCT between days 1 and 3. 1 - patients who registered an increasing or a minor decreasing $(<5 \%$ of the value at 24 hours) of serum biomarkers; 2 - patients who registered a decreasing of serum biomarkers between 5-20\%; 3 - patients who registered a decreasing of serum biomarkers between $20-50 \%$; 4 - patients who registered a major decreasing ( $>50 \%$ ) of serum biomarkers. NLCR, Neutrophil/ lymphocyte count ratio; FIB, fibrinogen; CRP, C-reactive protein; PCT, procalcitonin. 


\section{DISCUSSION}

In our study, the etiology of sepsis was identified in $46.7 \%$ of cases, similar to other data published in the medical literature $[14,15]$. In this context, monitoring the kinetics of inflammatory biomarkers in the first 4872 hours can be a useful method to assess the outcome of patients with sepsis, the response to antimicrobial therapy and the rates of mortality.

The increase of NLCR in sepsis is the result of a complex process which includes the response of the host immune system associated with neutrophilia and the apoptosis of lymphocytes in order to control the inflammatory response [16]. The role of NLCR in the early recognition and prediction of a poor outcome in sepsis was intensely studied in the last years. The results were controversial, because whereas some authors showed that NLCR was a suitable instrument in sepsis management [17], others concluded that NLCR was less reliable than CRP or PCT [18]. On the other hand, the most studies which assessed the kinetic patterns of NLCR were performed in patients with non-infectious causes for systemic inflammatory response syndrome (SIRS) [19]. Wasko et al. concluded that in the first five days after total hip or knee arthroplasty, NLCR levels achieved a peak level after 72 hours and the preoperative levels after 120 hours, faster than the time course of CRP [12]. Furthermore, in patients with malignancies receiving immunotherapy, NLCR levels decreased by 0.09 per month in patients who responded to therapy

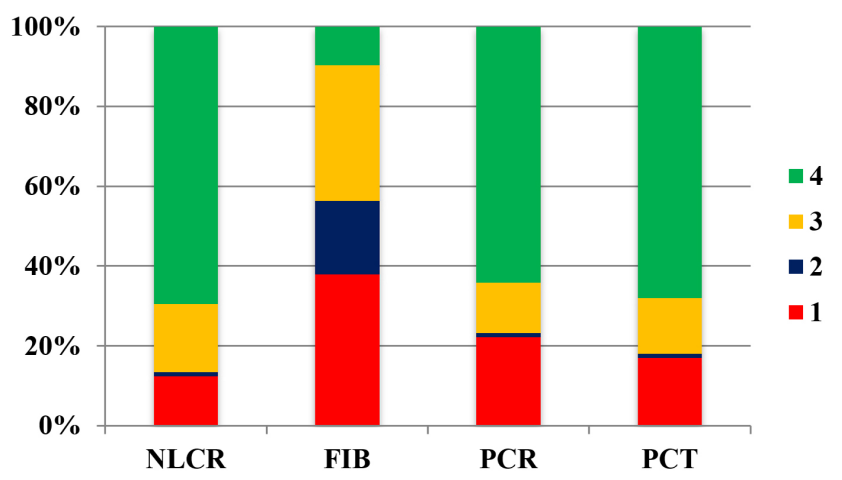

Fig. 4. Comparison of proportions of NLCR, FIB, CRP and PCT between days 0 and 3. 1 - patients who registered an increasing or a minor decreasing ( $<5 \%$ of the initial value) of serum biomarkers; 2 - patients who registered a decreasing of serum biomarkers between 5-20\%; 3 - patients who registered a decreasing of serum biomarkers between 20-50\%; 4 - patients who registered a major decreasing ( $>50 \%$ ) of serum biomarkers. NLCR, Neutrophil/lymphocyte count ratio; FIB, fibrinogen; CRP, C-reactive protein; $\mathrm{PCT}$, procalcitonin. in comparison with non-responders [20]. Holub et al. affirmed that NLCR had similar dynamics to PCT, due to the fast kinetics of WBC populations at the onset of inflammatory response [21]. In our study, NLCR values constantly decreased in time. The differences between NLCR levels at baseline, at 24 hours and at 72 hours were statistically significant. Moreover, in almost $43 \%$ of patients, NLCR decreased to one-half its initial levels after 24 hours. The percent was significantly higher in comparison with FIB $(\mathrm{p}<0.0001)$ and CRP $(p=0.0007)$. After 72 hours, in almost $68 \%$ of cases, the initial values of NLCR decreased with more than $50 \%$. The percent was lower in cases of FIB $(12 \%, \mathrm{p}<0.0001)$ and CRP $(58.2 \%, \mathrm{p}=0.2469)$ and higher in case of PCT (69.73\%, $\mathrm{p}=0.7694)$.

Plasma levels of FIB can increase or decrease in septic patients, depending on the stage of sepsis. Most cases are associated with high values of FIB, as an acute-phase protein which increases in presence of an inflammatory process. In the late stage of sepsis, reduced levels of FIB can be registered, due to its consumption [22]. Whereas FIB levels at admission are statistically significant correlated with a high mortality rate in sepsis [23], recent data concerning its kinetics in sepsis are limited, probably because the complex coagulation abnormalities responsible for FIB fluctuation. In the present study, FIB levels decreased between evaluations, but the difference between day 0 and day 1 was not statistically significant. On the other hand, only $1.58 \%$ of patients had a decreasing over $50 \%$ of the initial levels at 24 hours and only $12 \%$ at 72 hours.

CRP is an acute-phase reactant frequently used to predict inflammation, because its rapid increase after the aggression of a pathogen. Unfortunately, CRP levels remain elevated long time and its usefulness in monitoring the outcome is questionable. Moreover, the specificity of CRP for bacterial infections is under $50 \%$, while the area under the ROC curve is estimated at 0.68 [24]. Charles et al. performed a study on 180 patients with sepsis and observed that CRP time course in patients receiving potent empirical antibiotics was inappropriate. CRP values increased on days 2 and 3 and began to decrease on day 4, but with no statistical significance [25]. In our study, CRP levels increased on day 1 , but decreased statistically significant on day 3 . In comparison with NLCR and PCT, CRP had the slowest decreasing pattern. After 24 hours, only $16.12 \%$ of patients registered a decreasing of CRP of more than $50 \%$ of the initial values. 
Perhaps the most specific biomarker for sepsis diagnosis and the most reliable one in septic patients' monitoring is PCT, precursor of calcitonin, released by thyroid parafollicular cells and other neuroendocrine tissues such as hepatocytes, adipocytes, myocytes, in response to the presence of bacterial cytokines. The level of PCT in systemic circulation is under $0.1 \mathrm{ng} /$ $\mathrm{ml}$ in physiological conditions, but it increases within 4 hours in several bacterial infections and has a half-life between 22 and 26 hours [26]. In a study performed on 171 patients with sepsis or septic shock, Poddar et al. concluded that PCT level fall is related to a positive outcome. From day 0 to day 4, PCT level decreased from $3.48 \mathrm{ng} / \mathrm{ml}$ to $0.98 \mathrm{ng} / \mathrm{ml}$ in survivors group and increased from $5.27 \mathrm{ng} / \mathrm{ml}$ to $6.09 \mathrm{ng} / \mathrm{ml}$ in nonsurvivors [27]. On the other hand, Lipinska-Gediga et al. observed that PCT kinetics had significant prognostic value from day 3, as in the first 48 hours, the differences between evaluations did not have statistical significance (day 1 - day 2 difference, $\mathrm{p}=0.42$, day 2 - day 3 difference, $p=0.08$ ). The difference became significant between day 3 - day 5 . In the same study, the usefulness of PCT kinetics in appreciating sepsis prognosis was superior to CRP, but inferior to SOFA score [28]. In our study, PCT had a descendent kinetic pattern and the differences between samples were statistically significant. Almost $38 \%$ of patients registered a decreasing of more than $50 \%$ of the initial values of PCT after 24 hours and almost $70 \%$ after 72 hours.

The present study has several limitations. The first one consists in low number of patients with sepsis. Another limitation is that the enrollment was performed in a tertiary-care department of infectious diseases and included only mild or moderate cases of sepsis. Severe cases with life-threatening organ dysfunctions were admitted in the intensive care units and were not included in our research.

\section{CONCLUSIONS}

NLCR and PCT registered descendent kinetic patterns in the first 72 hours from admission, with statistically significance between assessments. In cases of NLCR and PCT, about $40 \%$ of patients had a decreasing of more than $50 \%$ of the initial values at 24 hours and about $70 \%$ at 72 hours. Conversely, FIB and CRP registered slower descendent kinetic patterns.

\section{ACKNOWLEDGEMENTS}

This work is part of "Carol Davila" University of Medicine and Pharmacy PhD program.

\section{AUTHORS' CONTRIBUTIONS}

All the authors had equally contributed in elaborating the paper.

\section{- CONFLICT OF INTEREST}

None to declare.

\section{REFERENCES}

1. Previsdomini $M$, Gini $M$, Cerutti $B$, Dolina $M$, Perren $A$. Predictors of positive blood cultures in critically ill patients: a retrospective evaluation. Croat Med J. 2012;53(1):30-39.

2. Phua J, Ngerng W, See K, Tay C, Kiong T, Lim H, et al. Characteristics and outcomes of culture-negative versus culture-positive severe sepsis. Crit Care. 2013;17(5):R202.

3. Kethireddy S, Bilgili B, Sees A, Kirchner H, Ofoma U, Light R, et al. Culture-Negative Septic Shock Compared With CulturePositive Septic Shock: A Retrospective Cohort Study. Crit Care Med. 2018;46(4):506-512.

4. Jenkins TC, Haukoos JS, Cotton E, Weitzenkamp D, Frank DN, Burman WJ. Time Course of C-Reactive Protein and Procalcitonin Levels During the Treatment of Acute Bacterial Skin Infections. Open Forum Infect Dis. 2018;5(3):ofy029.

5. Riedel S, Melendez JH, An AT, Rosenbaum JE, Zenilman JM. Procalcitonin as a marker for the detection of bacteremia and sepsis in the emergency department. Am J Clin Pathol. 2011;135(2):182-9.

6. Markanday A. Acute Phase Reactants in Infections: EvidenceBased Review and a Guide for Clinicians. Open Forum Infect Dis. 2015;2(3):ofv098.

7. Davidson J, Tong S, Hauck A, Lawson DS, da Cruz E, Kaufman J. Kinetics of procalcitonin and C-reactive protein and the relationship to postoperative infection in young infants undergoing cardiovascular surgery. Pediatr Res. 2013;74(4):413-419.

8. Eschborn S, Weitkamp JH. Procalcitonin versus C-reactive protein: review of kinetics and performance for diagnosis of neonatal sepsis. J Perinatol. 2019;39(7):893-903.

9. Berends ET, Kuipers A, Ravesloot MM, Urbanus RT, Rooijakkers SH. Bacteria under stress by complement and coagulation. FEMS Microbiol Rev. 2014;38:1146-1171.

10. Thurnham DI, McCabe GP. Influence of infection and inflammation on biomarkers of nutritional status with an emphasis on vitamin A and iron. WHO report: Priorities in the assessment of vitamin A and iron status in populations. 
Available online at: www.jccm.ro

Geneva(Switzerland): World Health Organization, 2012.

11. Zahorec R. Ratio of neutrophil to lymphocyte counts-rapid and simple parameter of systemic inflammation and stress in critically ill. Bratisl Lek Listy. 2001;102(1):5-14.

12. Wasko MK, Struminski M, Bobecka-Wesolowska K, Kowalczewski J. Neutrophil-to-lymphocyte ratio shows faster changing kinetics than C-reactive protein after total hip and knee arthroplasty. J Orthop Translat. 2017;10:36-41.

13. Singer $M$, Deutschman CS, Seymour CW, Shankar-Hari M, Annane $D$, Bauer $M$, et al. The Third International Consensus Definitions for Sepsis and Septic Shock (Sepsis-3). JAMA. 2016;315(8):801-10.

14. Pedro TCS, Morcillo AM, Baracat ECE. Etiology and prognostic factors of sepsis among children and adolescents admitted to the intensive care unit. Rev. bras. ter. intensiva [online]. 2015;27(3):240-246.

15. Heffner AC, Horton JM, Marchick MR, Jones AE. Etiology of illness in patients with severe sepsis admitted to the hospital from the emergency department. Clin Infect Dis. 2010;50(6):814-820.

16. Liu X, Shen $Y$, Wang $H$, Ge $Q$, Fei A, Pan S. Prognostic Significance of Neutrophil-to-Lymphocyte Ratio in Patients with Sepsis: A Prospective Observational Study. Mediators Inflamm. 2016:8191254.

17. Ljungström $L$, Pernestig $A K$, Jacobsson $G$, Andersson $R$, Usener B, Tilevik D. Diagnostic accuracy of procalcitonin, neutrophil-lymphocyte count ratio, C-reactive protein, and lactate in patients with suspected bacterial sepsis. PLoS One. 2017;12(7):e0181704

18. Westerdijk K, Simons KS, Zegers M, Wever PC, Pickkers P, de Jager CPC. The value of the neutrophil-lymphocyte count ratio in the diagnosis of sepsis in patients admitted to the Intensive Care Unit: A retrospective cohort study. PLOS ONE 14(2):e0212861.
The Journal of Critical Care Medicine 2020;6(1) • 31

19. Yombi JC, Schwab PE, Thienpont E. Neutrophil-to-lymphocyte ratio (NLR) distribution shows a better kinetic pattern than C-reactive protein distribution for the follow-up of early inflammation after total knee arthroplasty. Knee Surg Sports Traumatol Arthrosc. 2016;24(10):3287-3292.

20. Ameratunga $M$, Chenard-Poirier M, Moreno Candilejo I, Pedregal M, Lui A, Dolling D, et al. Neutrophil-lymphocyte ratio kinetics in patients with advanced solid tumours on phase I trials of PD-1/PD-L1 inhibitors. Eur J Cancer. 2018;89:56-63.

21. Holub, M., Beran, O., Kaspríková, N, Chalupa P. Neutrophil to lymphocyte count ratio as a biomarker of bacterial infections. Cent Eur J Med. 2012;7(2):258-61.

22. Sharma A, Sikka M, Gomber S, Sharma S. Plasma Fibrinogen and D-dimer in Children With Sepsis: A Single-Center Experience. Iran J Pathol. 2018;13(2):272-275.

23. Azfar M, Khan M, Khurshid M. Fibrinogen at admission is an independent predictor of mortality in severe sepsis and septic shock. Crit Care. 2014;18(1):P209.

24. Pradhan S, Ghimire A, Bhattarai B, Khanal B, Pokharel K, Lamsal $\mathrm{M}$, et al. The role of $\mathrm{C}$-reactive protein as a diagnostic predictor of sepsis in a multidisciplinary Intensive Care Unit of a tertiary care center in Nepal. Indian J Crit Care Med. 2016;20(7):417-420.

25. Charles PE, Tinel C, Barbar S, Aho S, Prin S, Doise JM, et al. Procalcitonin kinetics within the first days of sepsis: relationship with the appropriateness of antibiotic therapy and the outcome. Crit Care. 2009;13(2):R38.

26. Davies J. Procalcitonin. J Clin Pathol. 2015;68(9):675-9.

27. Poddar B, Gurjar M, Singh S, Aggarwal A, Singh R, Azim A, et al. Procalcitonin kinetics as a prognostic marker in severe sepsis/ septic shock. Indian J Crit Care Med. 2015;19(3):140-146.

28. Lipińska-Gediga M, Mierzchała-Pasierb M, Durek G. Procalcitonin kinetics - prognostic and diagnostic significance in septic patients. Arch Med Sci. 2016:12(1):112-9. 